\title{
Die Industrie 4.0 aus ethischer Sicht
}

\author{
Oliver Bendel
}

Eingegangen: 25. März 2015 / Angenommen: 30. Juni 2015 / Online publiziert: 23. Juli 2015

(C) Springer Fachmedien Wiesbaden 2015

Zusammenfassung Der vorliegende Beitrag arbeitet die wesentlichen Merkmale der Industrie 4.0 heraus und setzt sie ins Verhältnis zur Ethik. Es interessieren vor allem Bereichsethiken wie Informations-, Technik- und Wirtschaftsethik. Am Rande wird auf die Maschinenethik eingegangen, im Zusammenhang mit der sozialen Robotik. Es zeigt sich, dass die Industrie 4.0 neben ihren Chancen, die u. a. ökonomische und technische Aspekte betreffen, auch Risiken beinhaltet, denen rechtzeitig in Wort und Tat begegnet werden muss.

Schlüsselwörter Industrie 4.0 Cyber-physische Systeme $\cdot$ Automatisierung · Produktion $\cdot$ Ethik $\cdot$ Informationsethik

\begin{abstract}
This article highlights the essential features of the industry 4.0 and puts them in relation to ethics. Of special interest are the fields of applied ethics such as information, technology and business ethics. Machine ethics is mentioned in passing in connection with social robotics. It is evident that the industry 4.0 in addition to opportunities, affecting among other things economic and technical aspects, includes also risks which must be addressed in word and deed in a timely manner.
\end{abstract}

Keywords Industry $4.0 \cdot$ Cyber-physical systems · Automation · Production Ethics $\cdot$ Information ethics

O. Bendel $(\square)$

Hochschule für Wirtschaft, Institut für Wirtschaftsinformatik,

Fachhochschule Nordwestschweiz,

Bahnhofstrasse 6,

5210 Windisch, Schweiz

E-Mail: oliver.bendel@fhnw.ch 


\section{Wesentliche Merkmale der Industrie 4.0}

„Industrie 4.0“ ist ein Marketingbegriff, der für ein strategisches Projekt der deutschen Bundesregierung steht. Die Nummer verweist auf die vierte industrielle Revolution. Es handelt sich um kein Release wie bei einer Software. Man denkt an „Web 2.0“ und ,Web 3.0“, wo ebenfalls bestimmte Vorstellungen und Bewertungen vermittelt werden, und an die damit angesprochenen Entwicklungen, die mit der Industrie 4.0 durchaus zu tun haben (vgl. Bendel 2015). Für die Wirtschaftsinformatik ist die Industrie 4.0 ein wichtiges Forschungs- und Anwendungsfeld geworden (vgl. Herda und Ruf 2014).

Charakteristisch für die Industrie 4.0 sind Automatisierung, Autonomisierung, Flexibilisierung und Individualisierung in der Digitalisierung, wobei eine möglichst vollständige Vernetzung sowie die Erhöhung von Effektivität und Effizienz angestrebt werden (vgl. Bendel 2014a). Kern ist die Smart Factory, die intelligente Fabrik. Diese wird mit Hilfe von cyber-physischen Systemen (die aus physischen Komponenten bestehen, virtuelle Inputs erhalten und physische Produkte hervorbringen) und innovativen Industrierobotern betrieben und ist mit ihrer Umwelt verbunden (vgl. Bauernhansl et al. 2014; Janiesch 2013). Im Folgenden wird auf die genannten Begrifflichkeiten eingegangen (vgl. Bendel 2015):

- Automatisierung ist ein altes Thema und ein weites Feld. Schon in der Antike wurden Apparaturen erfunden, die sich selbstständig in Bewegung setzten. Die Schweiz ist Automatenland, was u. a. Pierre Jaquet-Droz zu verdanken ist, der im 18. Jahrhundert die berühmten „Androiden“ konstruiert hat. Deutschland ist ein Geburtsland des Computers, durch Pioniere wie Konrad Zuse. Im Kontext der Industrie 4.0 geht es um eine automatisierte Produktion, die elektronisch gesteuert ist, um automatisierte Produktionsanlagen und um automatisierte Datenübertragungen, die wiederum die Produktion beeinflussen.

- Autonomie ist Selbstständigkeit von Maschinen und Menschen. In der Industrie 4.0 löst die eine immer mehr die andere ab. Im Zuge dieser Entwicklung, der Autonomisierung der Maschinen, rückt der Mensch ins zweite Glied. Er kontrolliert und wartet sie, die selbst zu entscheiden und zu handeln beginnen. Mehr und mehr autonom sind auch cyber-physische Systeme und Industrieroboter. Letztere verlassen ihre angestammten Plätze und mischen sich unter die Arbeiter, sofern noch welche anwesend sind. Serviceroboter, Flugdrohnen, Kraftfahrzeuge und Anlagen werden gleichfalls immer eigenständiger und transferieren permanent Daten an die intelligente Fabrik.

- Flexibilisierung ist gegeben, wenn just in time auf Anforderungen reagiert werden kann. Die Produktion der intelligenten Fabrik wird von der einen zur anderen Minute beschleunigt, verlangsamt, gestoppt, neu ausgerichtet oder angeordnet. Es werden andere Gegenstände in Serie hergestellt oder spezielle innerhalb der Serie, etwa mit Hilfe von 3D-Druckern. Eingebunden in die Wertschöpfungsprozesse sind Logistik- und Zulieferbetriebe und überhaupt Partnerfirmen, verwendet werden Daten aus sozialen Netzwerken, aus Informationssystemen und Datenbanken, von Verkaufsstellen, Messpunkten und aus dem Internet der Dinge (vgl. Sendler 2013, S. 10). 
- Individualisierung hängt in mancherlei Aspekten mit Flexibilisierung zusammen. Die Anforderungen sind z. B. Kundenwünsche, die sich auf Form, Funktion und Inhalt beziehen. Man wird über partizipative Medien involviert, sodass „Individualisierung“ den Einzelnen und eine Gruppe betreffen kann, und über andere digitale und traditionelle Kanäle. Crowdsourcing-Plattformen spielen ebenfalls eine Rolle. Es resultieren hybride Produkte, die nicht nur materiell zum Nachfrager passen, sondern auch ,virtuell“", in Bezug auf Service- und Versicherungsleistungen. Bei Einzelanfertigungen sind abermals 3D-Drucker im Einsatz.

Die Vernetzung in der Industrie 4.0 umfasst demnach Dinge, Systeme und Menschen, konkret Betriebsleiter, Angestellte, Arbeiter, Kunden etc. in Wertschöpfungsprozessen. Die Smart Factory ist das Herzstück und durch die systematische Verknüpfung der internen Anlagen gekennzeichnet, funktioniert aber nicht ohne die ebenso systematische Anbindung an externe Komponenten, die nähere und weitere Umwelt. Eine klassische industrielle Produktion können sich Deutschland, Österreich und Schweiz immer weniger leisten. Outsourcing und Offshoring sind eine Lösung. Eine andere ist der radikale Umbau der Industrie. Ziele müssen schneller und einfacher erreicht, Bedürfnisse besser befriedigt werden, bei gleichzeitiger Kostensenkung in ausgewählten Geschäftsprozessen.

Neben der Fabrikation gehören Mobilität, Gesundheit sowie Klima und Energie zu den wichtigsten Anwendungsfeldern der Industrie 4.0. Damit ist eine roboterbasierte Fahrzeugproduktion (die zur Smart Production zählt) genauso relevant wie die Weiterentwicklung von Fahrerassistenzsystemen und selbstständig fahrenden Autos, die - wie angedeutet - Daten sammeln und an Werkstätten und Hersteller schicken (vgl. Bendel 2015). Operations-, Pflege- und Therapieroboter ergänzen menschliche Fachkräfte. Sie sind außerordentlich präzise respektive ausdauernd und können rund um die Uhr benutzer- und vorgangsbezogene Daten auswerten. Das intelligente Stromnetz, das Smart Grid, revolutioniert das Energiemanagement und verbindet Energieversorger und -systeme. Ein wichtiges Element ist dabei der Smart Meter, der intelligente Stromzähler.

Insbesondere Konzerne und größere Maschinenunternehmen können die Investitionskosten stemmen, die Fabriken umwandeln, die Infrastruktur aufbauen und die Vermittlungs- und Endgeräte durchsetzen. Dabei ist die Bereitschaft des Konsumenten gefragt, der zum Produzenten wird, zum Prosumenten. Er muss aktiver als bisher sein, seine Wünsche besser artikulieren können und eine veränderte Umgebung, sei es bei der Arbeit, zu Hause oder auf der Straße, akzeptieren (vgl. Bendel 2015).

\section{Die Bereichsethiken und die Maschinenethik}

Die Ethik ist eine Disziplin der Philosophie und hat die Moral zum Gegenstand. Man nennt sie auch Moralphilosophie, in Abgrenzung zu Moraltheologie und Moralökonomie. In der empirischen Ethik beschreibt man die Moral, in der normativen arbeitet man an einem Rahmen zu ihrer Begründung und Verortung. Die Bereichs- oder Spezialethiken beziehen sich auf abgrenzbare Anwendungsbereiche. Beispiele sind Umwelt-, Bio-, Militär-, Technik-, Medien-, Wissenschafts-, Wirtschafts-, Politikund Rechtsethik. Institutionell verankert sind hauptsächlich Wirtschafts- und Medi- 
zinethik, mit einigen Instituten und Lehrstühlen im deutschsprachigen Raum. Sie gehen die Problemfelder mit ihrem spezifischen Wissen und ihren speziellen Begriffen an. Neben der Informationsethik werden in diesem Beitrag Technik-, Wirtschaftsund Umweltethik herausgegriffen. Zudem wird die Perspektive der Maschinenethik eingenommen (vgl. Bendel 2012a).

Die Informationsethik findet ihr „Material“ im Internet (vgl. Kuhlen 2004). Sie befasst sich ebenso mit Geräten wie Datenbrillen und Privatdrohnen. Nach Bendel (2012c) hat sie die Moral (in) der Informationsgesellschaft zum Gegenstand. Sie untersucht, wie sich deren Mitglieder, Informations- und Kommunikationstechnologien (IKT), Informationssysteme und digitale Medien anbietend und nutzend, in moralischer Hinsicht verhalten bzw. verhalten sollen. Unter ihren Begriff fallen Computer-, Netz- und Neue-Medien-Ethik (vgl. Bendel 2012b). Gelehrt wird sie u. a. innerhalb von Informationswissenschaft und Wirtschaftsinformatik.

Die Technikethik bezieht sich auf moralische Fragen der Geräte- und Werkzeugproduktion und des Technik- und Technologieeinsatzes. Es kann um Fahrzeuge oder Waffen ebenso gehen wie um Nanotechnologie oder Kernenergie. Es bietet sich also ein breites Spektrum dar, inzwischen ein so breites, dass die klare Abgrenzung des Gegenstandsbereichs in Gefahr sein könnte. Zur Wirtschafts- und zur Wissenschaftsethik werden enge Beziehungen unterhalten. In der Informationsgesellschaft ist die Technikethik zudem eng mit der Informationsethik verbunden, die jene mehr und mehr ersetzt.

Die Wirtschaftsethik hat die Moral der Wirtschaft und in der Wirtschaft zum Gegenstand. Dabei ist der Mensch im Blick, der produziert, handelt und führt bzw. ausführt (verschiedene Formen der Individualethik) sowie konsumiert (Konsumentenethik), und das Unternehmen, das Verantwortung gegenüber Mitarbeitern, Kunden und Umwelt trägt (Unternehmensethik als Institutionenethik). Zudem interessieren die moralischen Implikationen von Wirtschaftsprozessen und -systemen sowie von Globalisierung und Monopolisierung (Ordnungsethik). In der Informationsgesellschaft ist die Wirtschaftsethik eng mit der Informationsethik verzahnt.

Die Umweltethik rekurriert auf moralische Fragen beim Umgang mit der belebten (Pflanzen- und Tierethik) und unbelebten Umwelt des Menschen. Im engeren Sinne verstanden, beschäftigt sie sich mit dem Verhalten gegenüber natürlichen Dingen und dem Verbrauch natürlicher Ressourcen. Wenn sie nicht allein Menschen und Unternehmen als moralische Subjekte begreift, die auf die Umwelt einwirken, sondern auch Maschinen, muss sie sich mit der Maschinenethik verständigen. Die Umweltethik unterhält zur Wirtschaftsethik vielfältige Beziehungen. Im Normativen kann sie sich mit dem Umweltschutz treffen; anders als dieser macht sie aber keine Vorschriften und keine Aktionen.

Die Maschinenethik hat die Moral von Maschinen zum Gegenstand, vor allem von teilautonomen und autonomen Systemen wie Agenten, bestimmten Robotern bzw. Drohnen, Computern im automatisierten Handel und selbstständig fahrenden Autos (vgl. Anderson und Anderson 2011). Sie kann innerhalb von Informations- und Technikethik eingeordnet oder als Pendant zur Menschenethik angesehen werden. Die Moralfähigkeit von Maschinen wird kontrovers diskutiert und die Moral von Menschen in diesem Zusammenhang sowohl über- als auch unterschätzt (vgl. Bendel 2012a). 


\section{Die Industrie 4.0 in der Moral}

Die Industrie 4.0 birgt Chancen und Risiken. Vorteilhaft sind neben Effizienz- und Effektivitätsgewinn u. a. Anpassungs- und Wandlungsfähigkeit der Wirtschaft sowie Verbesserung der Arbeitsergonomie. Nachteilig ist, dass die komplexen Systeme und Strukturen anfällig sind. Die eigentlichen Probleme entstehen vielleicht dort, wo die Ökonomie ihrem ursprünglichen Anliegen, der Sicherung des Lebensunterhalts, nicht mehr nachkommen kann. Im Folgenden werden die Konzepte der Automatisierung, Autonomisierung, Flexibilisierung, Individualisierung sowie Vernetzung auf Bereichsethiken und Maschinenethik übertragen. Es werden jeweils diejenigen herausgegriffen, die in besonderer Weise von Belang sind.

\subsection{Informationsethik}

In der Industrie 4.0 wird Automatisierung mit Digitalisierung umgesetzt. Die Produktionsanlagen und die stationären und mobilen Roboter werden mit Hilfe von Computertechnologien gesteuert. Die Informationsethik fragt nach der Verlässlichkeit in dieser Hinsicht, nach der Sicherheit für den Menschen mit Blick auf die Programmierung. Auch die Haftung - etwa des Programmierers, des für den Support zuständigen Informatikers oder des Betriebsleiters - ist von Interesse, nicht nur im rechtlichen Sinne, sondern auch im moralischen.

Die Autonomisierung ist ebenfalls mit Digitalisierung verbunden und mit selbstständigen maschinellen Entscheidungen, wobei diese verschiedene Ebenen betreffen. Die Informationsethik untersucht die Folgen der Entscheidungen für Menschen. Auch hier wird nach der Sicherheit gefragt, aber auf eine spezielle Weise, etwa inwieweit die Entscheidungen der Maschinen für den Mitarbeiter gut sind und ob er Gefahren und Belastungen ausgesetzt ist. Weiter geht es um teilautonome oder autonome Entscheidungen, die den Kunden und überhaupt das Mitglied der Informationsgesellschaft betreffen.

Die Individualisierung kann Verluste bei der informationellen Autonomie zeitigen. Persönliche Daten des Kunden, die in der Industrie 4.0 massenhaft anfallen, werden bei der Produktion und bei Marketing und Vertrieb verwendet und möglicherweise weitergegeben (Hauptfleisch 2015). Zudem können evtl. Supportmitarbeiter darauf zugreifen und die Daten missbrauchen. Ein zentraler Aspekt ist, dass der Kunde noch mehr als bisher in Abläufe integriert und er als Prosument aktiver als früher wird und die Daten, mit denen er das Produkt mitbestimmt, viel über ihn und seine Vorlieben verraten.

Vernetzt werden Produktionsanlagen, cyber-physische Systeme, stationäre und mobile Roboter und Bestandteile des Internets der Dinge, zudem Datenbanken und Informationssysteme aller Art. Ein wichtiger Aspekt für die Informationsethik ist das Hacken der Systeme. Durch Vernetzung entsteht tendenziell eine größere Anfälligkeit, insofern mehr Schwachstellen und Einfallstore vorhanden sind. Das Hacken kann zur Übernahme der Systeme führen sowie informationelle Autonomie und Datenschutz betreffen (vgl. Kagermann et al. 2013, S. 51). 


\subsection{Technikethik}

Automatisierung bedeutet die Omnipräsenz der Technik. Diese dominiert die Fabrikhallen, reduziert die Interaktionen zwischen bzw. substituiert Menschen. Im Extremfall zieht sie Vereinsamung und Isolation nach sich. Sie beinhaltet auch die Erhöhung der Abhängigkeit von Technik. Diejenigen, die noch anwesend sind, sind in jedem Arbeitsschritt auf diese angewiesen. Wichtig ist die „Abwesenheit unvertretbarer Risiken und Gefahren für Menschen und Umgebung durch den Betrieb des Systems““ (Kagermann et al. 2013, S. 51) und die Herstellung von „Safety“ (Liggesmeyer und Trapp 2014).

Ein Problem bei der Individualisierung ist, dass Erfolge bei der Standardisierung technischer Elemente zerstört werden können. Haushalte bauen sich ihre eigenen Lösungen und setzen diese im Freundeskreis, in der Nachbarschaft oder in der Region durch, über das Internet sogar darüber hinaus. Zudem wird die Automatisierung und Autonomisierung im Haushalt weitergeführt, bei damit entstehender Abhängigkeit und Anfälligkeit. Zugleich wird durch andere Merkmale der Industrie 4.0 Standardisierung gewünscht und gefördert.

Vernetzung ermöglicht, wie angesprochen, das Hacken von Komponenten und Systemen. Dadurch kann jemand sowohl in die Systeme eindringen und diese über informationstechnische Komponenten übernehmen als auch die Technik manipulieren und ruinieren und damit die Produkte verändern oder verunmöglichen, mit dem Ergebnis gefährdeter oder enttäuschter Verbraucher. Neben den erheblichen Risiken gibt es zahlreiche Chancen, etwa wenn Autos und Serviceroboter an Fabriken Fehler zurückmelden und diese dadurch beheben helfen.

\subsection{Wirtschaftsethik}

Automatisierung impliziert in der Industrie 4.0 die Ersetzung menschlicher Arbeitskraft (vgl. Hirsch-Kreinsen 2014, S. 18). Betroffen sind mehrheitlich einfache Arbeiter, deren Handgriffe und Fingerfertigkeiten von Maschinen nachgeahmt werden können, während Informatik- und Führungskräfte zumindest in den ersten Phasen der Revolution unentbehrlich bleiben. Automatisierung impliziert aber genauso, dass Maschinen und Menschen in Arbeitszellen kooperieren, mit dem erwünschten Nebeneffekt körperlicher Entlastung und höherer Sicherheit. Generell ist zu berücksichtigen, dass auch Outsourcing und Offshoring massive Umwälzungen nach sich ziehen.

Die Autonomisierung im Kontext der Wirtschaftsethik ist mit der Frage verbunden, ob ökonomische Konsequenzen positiver oder negativer Art vorhanden sind. Die Smart Factory kann selbst Urteile fällen, die ihre Existenz gefährden, ihre mittelund langfristige Produktion, die Arbeitsplätze, die an ihr hängen. Zugleich kann sie sich durch schnell getroffene und umgesetzte Entscheidungen Wettbewerbsvorteile verschaffen. Ferner ist relevant, ob Geschäftsleitung und Management und überhaupt moralische Akteure im Unternehmen an Autonomie verlieren.

Flexibilisierung wird erreicht durch selbst entscheidende Maschinen, die mit anderen Systemen just in time Daten- und Informationsaustausch betreiben. Notwendig sind schnelle Anbindung und engmaschige Vernetzung, aber auch Zugang zu und 
Aneignung von hochwertiger und aktueller Information, um Marktumfeld und -entwicklung beurteilen zu können. Der digitale Graben kann dadurch größer werden. Wer Investitionen dieser Größenordnung tätigen kann, bestimmt Produktionsarten und -standorte. Staatliche Förderungen haben bis dato vor allem Konzerne erhalten (vgl. Zühlke 2014). Flexibilisierung kann zudem Flexibilität von Mitarbeitenden notwendig machen, bis hin zu Spontaneinsätzen, Überstunden und Nachtarbeit.

Ein besonderer Gesichtspunkt ist die Vernetzung im Algorithmic und High-frequency Trading (Rettberg 2010). Zum Teil geht es um das Ziel, Mikrosekunden einzusparen, für dessen Erreichung milliardenteure Verbindungen gebaut werden. Ein damit verknüpfter und bereits angesprochener Aspekt ist, wer an der Vernetzung partizipiert und wer nicht. Diese ermöglicht oder erleichtert, wie ausgeführt, das Eindringen in die Systeme. Angreifer können die Produktion stoppen oder verändern und dadurch betriebliche Ausfälle und finanzielle Schäden, mit Folgen für Betrieb und Mitarbeiterschaft, verursachen.

\subsection{Umweltethik}

Der Automatisierung geht die Produktion von Automaten voraus. Für diese braucht es Kunststoffe und Metalle verschiedener Art, für die Elektronikkomponenten u. a. Kobalt, Gold, Platin und Coltan (Columbit-Tantalit bzw. Niobit-Tantalit). Bei der Gewinnung werden z. T. Umwelt und Gesundheit von Arbeitern und Einwohnern zerstört. Zudem müssen die zum Teil sehr schweren Anlagen über weite Strecken transportiert werden, in Einzelteilen oder gesamt, und installiert werden, was wiederum Energie und mithin Lebensraum verbraucht.

Flexibilisierung und Individualisierung führen einerseits dazu, dass punkt- und personengenau Bedürfnisse erfüllt werden, andererseits dazu, dass Sonderwünsche ständig umgesetzt werden, dass kreative Anwandlungen in einen Ressourcenabbau und eine vielleicht nicht notwendige Produktionskette münden. Wenn der Kunde das Sagen hat, stehen seine Bedürfnisse im Vordergrund, nicht diejenigen einer nachhaltigen Umweltpolitik, und nicht immer kann man der Corporate Social Responsibility genügend Raum verschaffen. Ein mögliches Ergebnis ist, dass die Müllberge wachsen und noch mehr $-\mathrm{z}$. T. giftiger - Schrott entsorgt werden muss.

Vernetzung bedeutet das Aufbauen und Betreiben von Fest- und Funknetzen. Durch Telefon- und Freileitungsmasten sowie Mobilfunkantennen werden Landschaften zerstört und Strahlungen verursacht. Zudem weisen damit zusammenhängende Geräte elektromagnetische Felder auf, etwa Smartphones, über die man mit der Smart Factory bzw. den Mittlerdiensten kommuniziert. Kabel müssen im Boden oder durch Meere verlegt werden, wodurch Erdbewegungen notwendig sind sowie Strömungen und Strukturen beeinflusst werden. Das Internet der Dinge, das mit der Industrie 4.0 assoziiert ist, wird das dichteste Geflecht sein, das jemals die Erde überwuchert hat.

In diesem Zusammenhang kann die Tierethik involviert werden. Die zunehmende Automatisierung, Autonomisierung und Vernetzung geschieht lediglich zum Teil in der geschlossenen Fabrik. Es ist ja geradezu Merkmal der Smart Factory, dass sie über sich selbst hinauswächst. Daraus resultieren immer mehr Tier-Maschine-Beziehungen, etwa zwischen Wildtieren und Fahrerassistenzsystemen oder Windkraftan- 
lagen und zwischen Nutztieren und automatisierten Ställen samt Melkmaschinen, die mit Produktionsanlagen verbunden sind. Den damit verbundenen Fragen kann sich die Tierethik in Zusammenarbeit mit neuen Disziplinen wie der Tier-ComputerInteraktion und der Tier-Maschine-Interaktion widmen. Letztlich geht es um eine angemessene Einbeziehung und Berücksichtigung aller Lebewesen und eine verbesserte Gestaltung von Schnittstellen.

\subsection{Maschinenethik}

Autonomisierung beinhaltet maschinelle Entscheidungen, die moralische Konnotationen haben. Die Maschinenethik versucht den Produktionsanlagen, den stationären und mobilen Robotern und einzelnen Geräten wie 3D-Druckern moralische Fähigkeiten beizubringen (vgl. Bendel 2014b). Autonome Systeme können sich falsch entscheiden, entweder weil sie unpassende Regeln befolgen oder Situationen und Vorgänge unkorrekt interpretieren. Sie können Menschen verletzen und Unfälle verursachen (vgl. Kagermann et al. 2013, S. 51), was neben der Maschinenethik die soziale Robotik zu bekämpfen versucht. Dieser geht es vor allem um sozial verträgliche Maschinen, beispielsweise darum, dass diese adäquat reagieren und agieren, in den Arbeitszellen und auf ihrem Weg durch die Hallen.

\section{Erste Lösungsansätze und Handlungsempfehlungen}

Im letzten Kapitel wurde die eingangs erarbeitete Strukturierung der Industrie 4.0 auf ausgewählte Bereichsethiken und die Maschinenethik übertragen. Diese konnten mit ihren speziellen Kompetenzen auffällige Risiken herausarbeiten. Um Lösungsansätze und Handlungsempfehlungen skizzieren zu können, braucht es sie nicht mehr. Vielmehr erfolgt die Darstellung allgemein aus der angewandten Ethik heraus anhand der genannten Strukturierung. Dabei wird auf das direkt tangierte Wohl des Menschen fokussiert:

- Im Kontext der Automatisierung ist die Sicherheit der Menschen zu gewährleisten, im Sinne der Haftungs- und der Lebenssicherheit. Strenge Sicherheitsrichtlinien und regelmäßige Überprüfungen können hierbei helfen, ebenso Erkenntnisse der sozialen Robotik. Bezüglich der Haftungsfragen ist mit Philosophen und Juristen zusammenzuarbeiten. Arbeiter sind nach Möglichkeit für höhere Aufgaben (etwa das Monitoring oder die Konzeption der Automation) umzuschulen.

- Was die Autonomisierung angeht, müssen die Entscheidungen der Maschinen, sofern sie Auswirkungen auf Menschen und deren Arbeitsplätze haben, fortwährend in Frage gestellt und gegebenenfalls durch Änderungen des Regelsatzes oder der Fallsammlung angepasst werden. Das Management der Smart Factory muss sich in dieser Thematik mit Erkenntnissen der Maschinenethik und der KI vertraut machen und Kooperationen mit Hochschulen und Forschungseinrichtungen eingehen.

- Mit Blick auf die Flexibilisierung ist eine gewisse Flexibilität der Mitarbeitenden vorauszusetzen; diese sind aber vor Missbrauch und Ausbeutung zu schützen. So 
müssen Ruhezeiten eingehalten werden, Nachtarbeit ist streng zu regeln, ebenso die Verfügbarkeit am Feierabend. Trends kann durchaus auch kritisch begegnet werden, und Verantwortliche müssen entscheiden, ob die Smart Factory bloße Erfüllungsgehilfin der Märkte sein soll. Auf Gewerkschaften kommen in diesem Zusammenhang neue Aufgaben zu.

- In Bezug auf die Individualisierung sind die persönlichen Daten von Kunden zu schützen, etwa durch Verschlüsselung und Anonymisierung. Eigenlösungen von Bürgern werden soweit wie möglich unterstützt, indem neue Standards entwickelt werden; mindestens werden sie auf die Gefahren hingewiesen. Durch Rückkanäle kann versucht werden, auf die Wünsche der Kunden Einfluss zu nehmen und sie für Konsumentenethik und Umweltschutz zu sensibilisieren. Ein gutes Produkt kann auch eines sein, das nur erdacht und nicht verwirklicht wird.

- Die Vernetzung von Menschen, Systemen und Dingen ist ständig zu überprüfen und teilweise wieder aufzulösen, etwa wenn Hacker an persönliche Daten gelangen, das eigene Auto übernehmen oder man sich durch zu viele Funkmasten und Accesspoints gestört und beeinträchtigt fühlt. Es muss regelmäßig nach den Wünschen und Bedürfnissen der Mitarbeitenden und Kunden gefragt werden, z. B. mit Hilfe von Umfragen, und es sollte eine möglichst große Informationstransparenz vorhanden sein, sodass man als Betroffener gegebenenfalls reagieren kann.

Es braucht demnach eine Reihe von Maßnahmen und Wegleitungen. Diese können durch Moralkodizes und die Arbeit von Ethikkommissionen sowie weitere Instrumente der angewandten Ethik ergänzt werden. Es wurde deutlich, dass die Lösungsansätze und Handlungsempfehlungen eine informationelle, rechtliche, technische und wirtschaftliche Absicherung beinhalten. Dabei sind nicht zuletzt Informatik und Wirtschaftsinformatik gefordert.

\section{Ein gutes, glückliches Leben}

Als Marketingbegriff entzieht sich „Industrie 4.0“ ein Stück weit einer wissenschaftlichen Präzisierung (vgl. Bendel 2014a). Die Frage ist, was man zur Industrie zählt, was man als Industrialisierung bezeichnet und ob diese ein wertendes Konzept bedeutet. Vorteilhaft sind u. a. Anpassungs- und Wandlungsfähigkeit, Ressourceneffizienz, Verbesserung von Ergonomie und Erhöhung von (bestimmten Formen der) Sicherheit. Nachteilig ist, dass die komplexen Strukturen der Industrie 4.0 hochgradig anfällig sind und Mensch und Umwelt in ihrer Existenz gefährdet werden können.

Die Bereichsethiken sind gefragt, um nicht nur Risiken aufzudecken, sondern auch Vorschläge zu unterbreiten für ein gutes, glückliches sowie gesundes Leben. Dabei besteht ein solches für die Mehrheit kaum in der Technologiefreiheit, obwohl diese für bestimmte Bereiche durchaus zu diskutieren ist (vgl. Bendel 2012d). Vielmehr geht es darum, einerseits Innovations- und Wettbewerbsfähigkeit sowie Sicherheit an der Werkbank zu gewährleisten, andererseits den Menschen Beschäftigungsund Entfaltungsmöglichkeiten zu bieten und sie nicht von Maschinen an den Rand drängen zu lassen. Die angewandte Ethik hat mit der Industrie 4.0 ein neues, großes Untersuchungsgebiet gefunden. 


\section{Literatur}

Anderson M, Anderson SL (Hrsg) (2011) Machine ethics. Cambridge University Press, Cambridge

Bauernhansl T, ten Hompel M, Vogel-Heuser B (2014) Industrie 4.0 in Produktion, Automatisierung und Logistik. Springer Vieweg, Wiesbaden

Bendel O (2012a) Maschinenethik. Gabler Wirtschaftslexikon, Gabler/Springer, Wiesbaden. http:/wirtschaftslexikon.gabler.de/Definition/maschinenethik.html. Zugegriffen: 23. Juni 2015

Bendel O (2012b) Informationsethik. Gabler Wirtschaftslexikon, Gabler/Springer, Wiesbaden. http://wirtschaftslexikon.gabler.de/Definition/informationsethik.html. Zugegriffen: 23. Juni 2015

Bendel O (2012c) Die Medizin in der Moral der Informationsgesellschaft. IT Health 3(2):17-18

Bendel O (2012d) Die Rache der Nerds. UVK, Konstanz

Bendel O (2014a) Industrie 4.0. Gabler Wirtschaftslexikon, Gabler/Springer, Wiesbaden. http://wirtschaftslexikon.gabler.de/Definition/industrie-4-0.html. Zugegriffen: 23. Juni 2015

Bendel O (2014b) Maschinenethik in der Industrie 4.0: Plädoyer für einfache moralische Maschinen. In: Wissenschaftsjahr 2014 - Die Digitale Gesellschaft, 12. Juni 2014. http://www.digital-ist.de/experten-blog/maschinenethik-in-der-industrie-40.html. Zugegriffen: 23. Juni 2015

Bendel O (2015) Chancen und Risiken 4.0. UnternehmerZeitung 2(21):35-35

Hauptfleisch K (2015) Über Machine-to-Machine und Internet der Dinge zur Industrie 4.0. Computerwoche, 10. März 2015. http://www.computerwoche.de/a/ueber-machine-to-machine-und-internet-derdinge-zur-industrie-4-0,3068010. Zugegriffen: 23. Juni 2015

Herda N, Ruf S (2014) Industrie 4.0 aus der Perspektive der Wirtschaftsinformatik. Wirtschaftsinformatik Manag 5/2014:7-19

Hirsch-Kreinsen H (2014) Wandel von Produktionsarbeit - „Industrie 4.0“. Soziologisches Arbeitspapier Nr. 38/2014. http://www.wiso.tu-dortmund.de/wiso/is/de/forschung/soz_arbeitspapiere/Arbeitspapier_Industrie_4_0.pdf. Zugegriffen: 23. Juni 2015

Janiesch $\bar{C}$ (2013) $\bar{C}$ yber-physische Systeme. In: Kurbel K, Becker J, Gronau N et al (Hrsg) Enzyklopädie der Wirtschaftsinformatik, 8. Aufl. Oldenbourg, München. http://www.enzyklopaedie-der-wirtschaftsinformatik.de. Zugegriffen: 23. Juni 2015

Kagermann H, Wahlster W, Helbig J (Hrsg) (2013) Umsetzungsempfehlungen für das Zukunftsprojekt Industrie 4.0: Abschlussbericht des Arbeitskreises Industrie 4.0. April 2013. http://www.bmbf.de/ pubRD/Umsetzungsempfehlungen_Industrie4_0.pdf. Zugegriffen: 23. Juni 2015

Kuhlen R (2004) Informationsethik: Umgang mit Wissen und Informationen in elektronischen Räumen. UVK, Konstanz

Liggesmeyer P, Trapp M (2014) Safety: Herausforderungen und Lösungsansätze. In: Bauernhansl T, ten Hompel M, Vogel-Heuser B (Hrsg) Industrie 4.0 in Produktion, Automatisierung und Logistik. Springer Vieweg, Wiesbaden, S 433-449

Rettberg U (2010) An der Börse geht es um Nanosekunden. Handelsblatt, 10. Mai 2010. http://www. handelsblatt.com/finanzen/maerkte/boerse-inside/handelssysteme-an-der-boerse-geht-es-um-nanosekunden/3432762.html. Zugegriffen: 23. Juni 2015

Sendler U (2013) Industrie 4.0- Beherrschung der industriellen Komplexität mit SysLM (Systems Lifecycle Management). In: Sendler U (Hrsg) Industrie 4.0 - Beherrschung der industriellen Komplexität mit SysLM. Springer Vieweg, Wiesbaden, S 1-19

Zühlke K (2014) Industrie-4.0-Förderung fast nur für die Großen. elektroniknet.de, 6. August 2014. http:// www.elektroniknet.de/elektronikfertigung/strategien-trends/artikel/111743/. Zugegriffen: 23. Juni 2015 